\title{
Une démarche de prévention continue dans le domaine de la radiographie industrielle
}

Compte tenu de la haute activité des sources de rayonnements ionisants mises en œuvre, la radiographie industrielle représente pour les travailleurs ainsi que pour le public un risque majeur qu'il convient de prévenir et de limiter.

Comme en témoignent au niveau mondial les derniers accidents survenus, tels que par exemple ceux observés au Chili en 2005, à Dakar et Abidjan en 2006, en Tunisie en 2008, les conséquences de ceux-ci sur la santé peuvent s'avérer dramatiques.

Si la France n'a pas connu, fort heureusement, d'accident d'une telle ampleur, grâce notamment à l'application de mesures de prévention spécifiques à cette activité professionnelle, il n'en demeure pas moins que les risques liés à l'utilisation des appareils de radiographie restent majeurs, notamment sur chantier où la coactivité des entreprises et la proximité possible du public complexifient la mise en œuvre des mesures de prévention.

À ce titre, cette activité constitue un enjeu prioritaire en matière de radioprotection pour l'Autorité de sûreté nucléaire (ASN) et le ministère chargé du travail qui ont fixé, chacun dans leur domaine de compétence, des règles de protection renforcées pour cette activité professionnelle et exercent des contrôles réguliers de leur application.

Outre les actions conjointes de prévention auprès des entreprises engagées dans plusieurs régions (PACA, Haute Normandie...) en collaboration avec les CRAM, permettant l'élaboration de chartes de bonnes pratiques adaptées aux spécificités techniques, économiques et sociales locales, l'ASN a mené une action de prévention d'envergure nationale.

Celle-ci, engagée lors des journées du Comité français des essais non destructifs (COFREND) organisées à Beaune du 24 au 26 mai 2005, visait à inciter les professionnels concernés d'une part, à définir, au niveau national, des règles de bonnes pratiques, notamment pour la préparation et le déroulement des chantiers et d'autre part, à engager une réflexion sur la justification de l'activité de radiographie industrielle. Cette proposition ayant reçu un accueil favorable des participants, l'ASN a formalisé cette proposition par courrier en date du 15 juillet 2005 en suggérant à la COFREND de mettre en place et d'animer des groupes de travail sur ces questions. 
Afin de garantir la pertinence de ce travail, le COFREND s'est engagé dans cette démarche en s'appuyant, pour ce qui concerne le volet « bonnes pratiques », sur la compétence d'experts de la Société française de radioprotection (SFRP). Compte tenu de l'ampleur de l'action, neuf groupes de travail ont été constitués, chacun chargé d'une thématique propre telle que la formation, le matériel, l'analyse de poste, le retour d'expérience, etc.

Les recommandations de ces neuf groupes de travail sont présentées dans cette publication.

Pour s'assurer de la cohérence des démarches nationale et régionale engagées, évaluer leur impact en matière de santé et sécurité et, enfin, veiller à leur mise à jour compte tenu notamment des évolutions techniques et réglementaires, la direction générale du travail (DGT) conjointement avec l'ASN constituera avant la fin de l'année 2008 un comité de suivi regroupant les principaux acteurs de la prévention dans le domaine de la radiologie industrielle.

A.C. Lacoste, Président de l'Autorité de sûreté nucléaire

J.-D. Combrexelle, Directeur général du travail 